COGNitive STUdies | Études COGNITIVES, 14: 131-147 SOW Publishing House, Warsaw 2014

DOI : $10.11649 / \mathrm{cs} .2014 .012$

MARIA H. GOLKA

Adam Mickiewicz University, Poznan, Poland

maria.golka@amu.edu.pl

\title{
LA CATÉGORISATION LINGUISTIQUE DES COULEURS : NIVEAUX D'ÉLÉMENTARITÉ DES NOMS DE COULEURS FRANÇAIS
}

\begin{abstract}
Linguistic Categorisation of Colours: Relative Basicness of French Colour Terms. Categorisation is one of the basic cognitive processes. Because of the continuous character of the colour spectrum, colour terms constitute an interesting material to study linguistic categorisation. The first part of this article offers a review of research on the linguistic categorisation of colours, especially on basic colour terms and prototypes. In the second part, the results of an empirical study on the basicness of French colour terms will be presented. The results suggest that colour terms are categorised as prototypically structured concepts, which supports the hypothesis of the relative basicness of colour terms.
\end{abstract}

Keywords: linguistic categorisation, basic colour terms, relative basicness, semantics.

\section{Introduction}

La catégorisation est l'un des processus cognitifs les plus fondamentaux. Pour penser, pour manier les concepts, pour parler, il faut diviser le monde en unités. La catégorisation dite conceptuelle qui se passe constamment dans nos têtes n'est pas consciente et donc elle est difficilement accessible à la description scientifique. En revanche, la catégorisation linguistique nous offre tout un éventail de possibilités de recherches. La langue morcelle la réalité en unités commodes à communiquer. La structure de ces unités, des concepts, peut nous donner des informations sur notre façon de percevoir le monde.

La catégorisation, elle-même, est un processus difficile à observer. Mais la catégorisation de certains concepts flous est d'autant plus impénétrable qu'il est difficile d'observer les frontières de ces unités dans le monde réel.

Tel est le cas de la catégorisation des couleurs. Le spectre des couleurs est un continuum où il est difficile d'apercevoir des limites entre des unités. Pourtant, la 
plupart des langues possèdent les dénominations pour des couleurs particulières. Certains d'entre ces noms sont plus importants, plus fréquemment utilisés; bref, plus commodes à communiquer. Mais est-ce que cette primauté de certains noms de couleurs dérive de la façon dont on perçoit le monde ou peut-être elle est totalement arbitraire? Peut-être à l'inverse : la façon de percevoir les couleurs est influencée par la langue qu'on utilise? De toute façon, on ne peut pas nier la constatation suivante :

«Le monde n'est donc pas une réalité objective existant en et de par elle-même. Il nous apparaît toujours d'une façon ou d'une autre par le biais de notre activité qui consiste à catégoriser sur base de notre perception, de nos connaissances, de notre état d'esprit; bref, à partir de notre condition humaine.» (Delbecque, 2002, p. 32).

Si l'on observe la terminologie des couleurs, on aperçoit sans peine que certains noms de couleurs ont le statut plus privilégié que les autres. Ces noms de couleurs élémentaires constituent-ils une classe distincte perceptuellement et conceptuellement? Ou bien, la catégorie des noms de couleurs a une structure prototypique, avec quelques représentants centraux et tout un éventail des exemplaires périphériques? C'est une question qui mérite d'être posée : est-ce que le caractère élémentaire de certains noms de couleurs français est un trait qui peut être décrit en termes des conditions nécessaires et suffisantes, ou bien cette propriété possède une structure prototypique?

\section{Les noms de couleurs élémentaires et la notion de prototype}

En 1969, le livre intitulé «Basic color terms : their universality and evolution», écrit par deux anthropologues et linguistes américains, Brent Berlin et Paul Kay a été publié. La publication du livre a été précédée de vastes recherches menées sur le matériel provenant de 98 langues. Ce travail vise à démontrer qu'il existe l'universalité dans la dénomination de couleurs à travers le monde entier.

La première des découvertes de Berlin et Kay est le dégagement de la liste de noms de couleurs élémentaires (basic color terms) communs à toutes les langues. L'inventaire de noms de couleurs élémentaires contient onze noms : blanc, noir, rouge, jaune, vert, bleu, brun, gris, orange, violet, rose. C'est-à-dire, la langue peut posséder au maximum onze noms de couleurs élémentaires. La prédominance de ces noms de couleurs par rapport aux autres réside dans quelques facteurs énumérés par les auteurs. Premièrement, la couleur à laquelle le mot faire référence ne peut pas être contenue dans une autre couleur. Par exemple pourpre est contenu dans le mot rouge donc il n'est pas un nom de couleur élémentaire. Deuxièmement, la construction du nom élémentaire est simple; il ne peut pas se composer de plus que d'un seul morphème. Rouge brique ou sang de boeuf ne sont pas des noms élémentaires, ainsi que rougeâtre (à cause du suffixe -âtre). Troisièmement, le nom de couleur élémentaire doit avoir une vaste étendue sémantique. Par exemple le nom blond, ne se référant qu'à la couleur des cheveux, est trop restreint pour pouvoir appartenir aux noms élémentaires. Quatrièmement, ils sont fréquemment utilisés, communs et connus de tous.

La deuxième découverte importante de Berlin et Kay c'est la hiérarchie des langues en fonction du nombre de noms de couleurs élémentaires qu'elles possèdent. 
Chaque langue possède un nombre limité de noms de couleurs élémentaires. C'est Berlin et Kay qui ont aperçu que les noms élémentaires n'apparaissent pas dans les langues d'une façon imprévisible. Quand une langue n'a que deux noms de couleurs c'est le noir et le blanc. Quand une langue a trois noms de couleurs élémentaires, il s'agit de noir, blanc, rouge. Quand elle en a quatre la quatrième est soit le jaune, soit le vert. Le cinquième nom élémentaire c'est le jaune ou le vert, en fonction de ce qui a été précédant. Comme le sixième apparaît le bleu. Le septième c'est le brun. S'il s'agit de quatre derniers noms élémentaires jusqu'à onzième, ils sont choisis sans ordre établi parmi le gris, le rose, l'orange et le violet.

Distribution universelle des termes de couleurs ${ }^{1}$ :

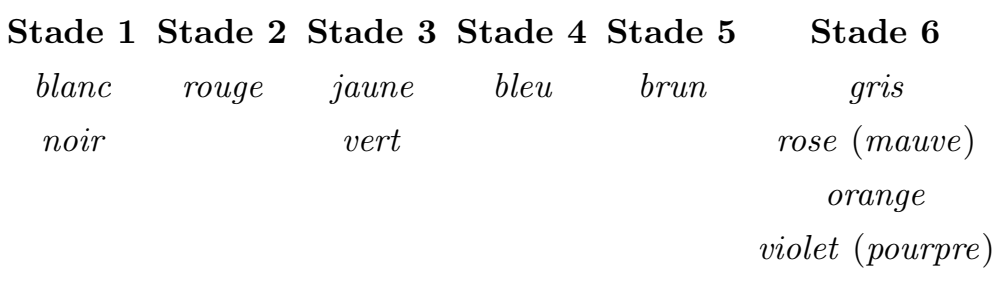

Cette hiérarchie de couleurs a été souvent niée par différents auteurs. Le fait que chaque langue possède au moins deux noms de couleur a été démenti par Anna Wierzbicka mais personne n'essaye même pas de contredire la primauté de l'opposition blanc/noir qui possède aussi la valeur quantitative et non seulement qualitative comme tous les autres termes de couleur.

La troisième chose importante décrite par Berlin et Kay c'est l'existence des couleurs focales (focal colors). Il s'agit des membres centraux, des exemples les plus parfaits désignés par les noms de couleurs élémentaires. Autrement dit, la couleur focale c'est une nuance de la couleur considérée par les utilisateurs d'une langue comme le meilleur exemple de cette couleur. On peut donc appeler la couleur focale le prototype, le meilleur représentant d'une couleur désignée par un nom donné.

Les découvertes plutôt théorétiques de Berlin et Kay ont reçu une confirmation empirique dans les recherches de Eleanor Rosch, menées au début des années 70. Rosch (encore sous son nom de jeune fille, Heider) a fait une série de quatre expériences concernant l'universalité dans la mémorisation et dénomination des couleurs.

Les résultats montrent qu'il existe une grande convergence des couleurs focales entre les langues. En outre, les couleurs focales se caractérisent par la saturation plus marquée que les autres nuances. Dans la plupart des langues, les noms des couleurs focales se sont avérés plus courts que ceux des autres couleurs, ce qui suggère une meilleure codabilité linguistique. On peut donc constater que les couleurs focales sont perceptuellement préférées par l'appareil cognitif. En plus, l'existence du nom de couleur dans la langue maternelle facilite la mémorisation de cette couleur.

L'article de Rosch de 1972 où elle décrit ses quatre expériences sur les couleurs (Heider, 1972, p.10-20) a tracé la voie pour ses recherches concernant les

1. La traduction française du schéma original de Berlin et Kay d'après : Delbecque (2002, p. 299). 
prototypes. Pourtant elle ne commence à utiliser le terme prototype qu'un an plus tard. C'est alors que paraît son article où elle fait la distinction entre les catégories perceptuelles et sémantiques (Heider, 1973, pp.111-144). La couleur appartient bien sûr aux catégories perceptuelles, mais ce type de catégories contient également le contour ou la direction. Les catégories sémantiques sont plus fréquentes. Les concepts appartenant à chacun de ces deux types ont la structure prototypique mais la provenance des prototypes est différente. Dans le cas des catégories sémantiques, il existe beaucoup de facteurs qui décident du caractère prototypique d'un exemplaire donné, comme par exemple le fait qu'il est commun, fréquent ou qu'il est présent dans des situations différentes. Dans le cas des catégories perceptuelles, le caractère prototypique d'un exemplaire dérive du fonctionnement du notre système cognitif. Les couleurs focales, elles aussi, sont plus expressives, plus marquées, et grâce à cela elles obtiennent le statut des prototypes. Ce qui met en relief les couleurs focales par rapport aux autres couleurs ce sont les processus au niveau de la rétine.

Au début de ses recherches concernant les prototypes (c'est-à-dire au début des années 70), Rosch a utilisé la notion de prototype seulement à propos des couleurs focales. Pourtant, bientôt il s'est avéré que ce terme peut même mieux décrire d'autres catégories. Donc enfin, en développant sa fameuse théorie des prototypes, Eleanor Rosch s'est occupé des catégories sémantiques. Ce qui nous intéresse, c'està-dire les catégories perceptuelles, a été laissé de côté. Par contre, la théorie des prototypes, basée plutôt sur les catégories sémantiques, a eu beaucoup de succès. La conception fondée sur l'idée d'air de famille de Wittgenstein a été une grande révolution. Le remplacement du modèle traditionnel des conditions nécessaires et suffisantes d'Aristote par la conception des prototypes est appelé même parfois la révolution roschienne. Cette conception est très utile, en particulier dans la linguistique, car elle permet d'expliquer beaucoup de concepts qui avant ont été difficiles à définir. En plus, cette théorie prend en considération la façon dont on perçoit le monde; elle est plus proche de la réalité que la conception idéaliste d'Aristote. Tout cela fait que la notion de prototype est très souvent utilisée et commence à sortir du cadre de la sémantique et lexicologie.

Georges Kleiber dans son livre «Sémantique du prototype» fait l'éloge de la notion de prototype et son utilité dans la linguistique. En fait, c'est vrai que la linguistique cognitive s'en sert souvent. A ce point que certains linguistes abusent de cette notion. C'est à ces procédés que se réfère Anna Wierzbicka. La régulation de ce problème est apparemment importante pour elle car son livre (Wierzbicka, 1999) commence par le chapitre traitant des prototypes.

L'attitude de Wierzbicka envers la sémantique de prototype est très ambivalente. D'une part, elle tente d'abattre tous les mythes liés à cette notion. D'autre part, elle la développe en apportant sa contribution à la théorie du prototype. Wierzbicka constate que la notion de prototype est surexploitée par les linguistes. Le prototype sert du mot-clé qui ouvre toutes les portes, de sauvetage universel dans le cas des concepts difficiles à définir. Selon Wierzbicka, une telle surexploitation des explications à l'aide des prototypes nuit au développement de la linguistique. Même si les concepts sont flous en eux-mêmes, on devrait chercher à les définir d'une façon précise. Au lieu de cela, on les explique souvent par leur caractère flou, imprécis 
et prototypique. D'après Wierzbicka, les prototypes ne peuvent pas remplacer les recherches lexicologiques sérieuses. Mais d'autre part, elle souligne qu'ils peuvent aider à formuler des explications qui seraient plus proches de nos concepts mentaux (Wierzbicka, 1999, p. 48).

Pour illustrer cette thèse, Wierzbicka donne deux groups d'exemples. Le premier d'entre ces groupes contient les mots qui sont expliqués par les prototypes, mais dont l'explication pourrait s'en passer, où l'usage de la notion de prototype est un abus. Le deuxième groupe d'exemples montre que la notion de prototype peut s'avérer très utile dans certains cas (à condition d'être traitée comme un outil analytique et non pas un moyen universel pour éviter l'effort mental, comme l'écrit Wierzbicka $(1999$, p. 27)). Dans le premier groupe il y a des mots déjà expliqués par différents auteurs qui ont constaté que l'usage du concept de prototype est nécessaire pour le faire. Wierzbicka arrive à expliquer les mêmes mots sans recourir à ce moyen. En même temps, elle admet qu'il y a des mots dont les définitions ont besoin de cette solution qui est offerte par la notion de prototype, surtout complétée par les explications à l'aide des primes sémantiques. Elle donne quelques exemples des définitions où l'usage de l'idée du prototype est fondé.

Les exemples les plus importants, illustrant la nécessité du concept de prototype sont les noms de couleurs. Les explications des mots de couleur sont possibles seulement par l'intermédiaire de la notion de prototype. C'est lié à une autre constatation très importante de Wierzbicka : les noms de couleurs ne sont pas des concepts universaux. Contrairement à ce que disaient Berlin et Kay, il y a des langues qui ne possèdent aucun terme de couleur. Le concept de couleur est absent sur la liste des primes sémantiques, construite par l'équipe de Wierzbicka ${ }^{2}$. Comme les couleurs n'appartiennent pas aux indefinibilia, ils ne peuvent pas servir à expliquer d'autres termes. Pour échapper au cercle vicieux il faut donc éviter de définir un mot de couleur par un autre terme de couleur, comme on le fait souvent dans les dictionnaires. Il faut chercher d'autres moyens de définir les termes de couleurs.

Et c'est ici que Wierzbicka propose de recourir au prototype. Mais elle comprend cette notion différemment que le faisait Rosch. Pour Wierzbicka, le prototype ce n'est pas le meilleur exemple d'une couleur mais l'objet ou le phénomène naturel qui l'exemplifie le mieux. Donc par exemple, le prototype de la couleur bleue est le ciel, du vert - l'herbe, du blanc et noir - le jour et la nuit.

Wierzbicka, en expliquant l'absence de la couleur sur sa liste des primitifs sémantiques, critique l'approche universaliste de Rosch et de Berlin et Kay. Certes, la façon de percevoir des couleurs est universelle mais, comme le remarque Wierzbicka, le langage illustre notre conceptualisation du monde et non pas la façon exacte dont nous percevons ce monde. On ne peut pas lier le langage directement avec les processus au niveau neuronal car il illustre ce qui se passe dans notre esprit et non pas dans notre cerveau. Ce n'est pas seulement le fonctionnement de l'appareil perceptif et des structures cérébrales qui se reflète dans le langage mais aussi la culture et l'environnement de la vie. Comme la perception de couleurs est la même indépendamment de la culture, il y a une certaine universalité dans leur dénomina-

2. Pourtant, différents concepts de couleur sont présents sur d'autres listes des primitifs sémantiques, cf. Pinker (2005, pp.617-621) et Puppel (2001, p. 200). 
tion. Mais, comme les cultures ont les besoins différents dans le domaine des noms de couleurs, il y a aussi de grandes divergences dans les systèmes d'appellations des couleurs d'une langue à l'autre.

Comme on le voit, la notion de prototype comprise par Rosch et par Wierzbicka ont peu de commun. Pour la première les prototypes de couleurs sont les couleurs focales, pour la seconde - les objets avec lesquels on peut identifier le nom de couleur. Il faut remarquer que le concept du prototype a une structure bipartite. Même le mot se compose de deux éléments. Le préfixe proto- met l'accent sur le côté primitif, originaire. C'est visible dans la définition du Petit Robert : «proto: élément, du gr. prôtos «premier, primitif, rudimentaire»». Et visiblement, les prototypes au sens de Wierzbicka ont ces traits. Ce sont des objets primitivement illustrant les couleurs. D'ailleurs c'est d'eux que le plus souvent vient le nom de couleur. Par contre, la conception de Rosch n'a rien à voir avec cette primitivité. Le prototype pour Rosch, c'est plutôt le modèle idéal de la couleur. Et cela correspond au sens du second élément du mot prototype. On lit dans Le Petit Robert : «type : modèle idéal, déterminant la forme d'une série d'objets».

Même si Eleanor Rosch postule l'universalisme dans la dénomination des couleurs et Wierzbicka renverse cette hypothèse; même si Rosch se concentre sur le côté perceptuel et Wierzbicka sur le côté culturel, environnemental; même si elles comprennent la notion du prototype différemment, une chose est sûre : les deux auteurs ont prouvé que les noms de couleurs peuvent être décrites à l'aide du concept de prototype. Elles ont démontré que le prototype, sous la forme de l'exemplaire soit idéal soit primitif, est une notion nécessaire quand on parle de la catégorisation des concepts aussi flous que les couleurs.

La notion de prototype proposée par Wierzbicka a été employé pour les analyses des noms de couleurs polonais (Tokarski, 2004). Dans son livre, Tokarski porte sur l'identification des prototypes de couleurs existant dans la culture et langue polonaise. D'autres chercheurs utilisent la notion de prototype dans des recherches diachroniques (Czachorowska, 2009), contrastives (Pietrzak-Porwisz, 2006 ; Zura, 2004) et cognitives (Waszakowa, 2009).

\section{L'hypothèse : l'élémentarité relative des noms de couleurs}

Berlin et Kay dans leur livre «Basic color terms» ont présenté la liste des noms de couleurs élémentaires, contenant onze éléments. C'est la liste maximale des noms élémentaires; selon les auteurs les langues en contiennent au minimum deux unités (blanc et noir), au maximum onze. En général, les langues indoeuropéennes possèdent tout un éventail des noms élémentaires. La liste des noms élémentaires en français contient onze éléments : noir, blanc, rouge, jaune, vert, bleu, brun, gris, orange, violet, rose ${ }^{3}$.

S'il s'agit de leurs propriétés, Berlin et Kay énumèrent quatre conditions qu'un mot doit remplir pour être nommé «nom de couleur élémentaire». C'est l'indépendance sémantique (la signification du nom d'une couleur ne peut pas être comprise dans celle d'un autre terme), la simplicité morphologique, une large étendue sé-

3. Il y a des hésitations concernant le choix d'équivalents français pour les mots anglais purple (fr. pourpre/violet) et pink (rose/mauve), cf. Delbecque (2002, p. 299). 
mantique et la fréquence d'usage importante. Ces quatre critères constituent la condition sine qua non du nom de couleur élémentaire.

Pourtant, dans des cas incertains, Berlin et Kay donnent des conditions supplémentaires. Premièrement, on prend en considération la productivité dérivationnelle du nom. Le nom qui produit les dérivés construits de la même façon que ceux produits par les noms de couleurs élémentaires (p. ex. les adjectifs de couleur à suffixe -ish en anglais, en -âtre en francais) est aussi supposé être un nom élémentaire. Deuxièmement, s'il y a des hésitations concernant le statut d'un nom de couleur, on se demande si le sens d'un nom de couleur est le sens primaire du mot. Si c'est sa signification secondaire ou même plus négligeable, le caractère élémentaire d'un tel nom est douteux. Troisièmement, cela ne doit pas être un nom emprunté récemment. Il devrait se comporter d'une façon typique de la langue donnée, c'est-à-dire s'accorder comme le font les autres noms de couleurs élémentaires (dans le cas du français).

Quoique Berlin et Kay prennent en considération les cas incertains, où la constatation du caractère élémentaire ou non d'un nom est difficile, ils constatent que la valeur élémentaire des noms de couleurs est un trait discret. Le nom ne peut pas être un peu élémentaire ou partiellement élémentaire. Le nom de couleur est soit élémentaire (onze cas), soit non. Les traits spécifiques des noms élémentaires font d'eux une classe séparée dont le statut diffère totalement de celui des autres noms de couleurs.

Par contre certains auteurs, tout en appréciant la liste de Berlin et Kay comme un apport important dans la science, trouvent que le caractère élémentaire des noms de couleur n'est pas un trait stricte et discret mais plutôt continu. Seija Kerttula qui a fait une vaste étude des termes de couleur anglais constate que chaque nom de couleur au moment donné se trouve à une certaine étape de son développement, de son enracinement dans la langue. C'est pour cette raison qu'elle rejette la conception de l'élémentarité stricte de Berlin et Kay en faveur de l'élémentarité relative (relative basicness) (cf. Kerttula, 2002, p. 84). D'après Kerttula, la frontière entre les noms de couleurs élémentaires et non élémentaires est floue. Certains d'entre les noms de couleurs sont élémentaires par excellence, certains n'ont pas ce statut, mais il y a encore tout un amas des cas intermédiaires. Si cette conception de l'élémentarité est juste, les traits qui décident du caractère élémentaire de certains noms devraient se répartir d'une façon continue et non pas discrète.

\section{Comment vérifier le niveau de l'élémentarité relative?}

\subsection{La méthode}

Pour vérifier le niveau de l'élémentarité des noms de couleurs particuliers il faut mesurer l'intensité des traits énumérés par Berlin et Kay comme les critères de l'élémentarité. Pour examiner si la conception relative de l'élémentarité est valable pour la dénomination française des couleurs il faut trouver la mesure qui permettra de comparer le niveau de relativité des uns par rapport aux autres.

Dans cette étude de l'élémentarité relative des termes de couleur français on s'est inspiré de la méthode élaborée par Seija Kerttula pour les noms de couleurs anglais. La méthode consiste à évaluer chaque nom de couleur sous différents angles afin de 
constater quel est son niveau relatif d'élémentarité. Pour chaque critère rempli par le nom, Kerttula lui attribue les points. Puis, elle compare le nombre de points de différents noms de couleurs pour pouvoir les ranger par l'ordre de leur niveau de l'élémentarité.

Ici on a transformé un peu les critères de Kerttula, en les accommodant aux conditions de la langue française (l'accord des adjectifs qui n'est pas présent en anglais), en supprimant certains critères ou en en ajoutant d'autres. Les critères qui ont servi de mesures pour le niveau de l'élémentarité des noms de couleurs français sont comme suit : la productivité dérivationnelle, la fréquence de l'usage, l'indépendance sémantique, la simplicité morphologique, l'accord et l'étendue sémantique.

Enfin, il faut souligner que les mesures prises ici n'ont pas un caractère absolu. Les valeurs attribuées aux noms de couleurs sous la forme des points ne peuvent que servir d'information sur leur niveau d'élémentarité relative. Ils peuvent servir à comparer les noms entre eux sans pourtant la possibilité de référence extérieure.

\subsection{Le choix de termes à étudier}

Comme les dimensions de ce travail ne permettent pas d'étudier tous les noms de couleurs fonctionnant en français ${ }^{4}$, on a dû se contenter d'en examiner quelquesuns. Au-delà de onze noms élémentaires de Berlin et Kay qu'on a jugés obligatoires à étudier, le choix était fortuit. C'était le tirage au sort parmi les termes du champ sémantique du rouge qui a décidé de la version finale du matériel analysé. La quinzaine de noms de couleurs qui vont être analysés est comme suit : noir, blanc, rouge, jaune, vert, bleu, brun, gris, orange, violet, rose, pourpre, queue-de-renard, rubicond, grenat.

\section{La vérification de l'hypothèse de l'élémentarité relative. Analyse des noms de couleurs selon les critères préétablis}

\subsection{La productivité dérivationnelle}

Ce critère évalue le niveau d'enracinement d'un nom dans la langue. En général, les mots existant depuis longtemps ont beaucoup de dérivés. Plus le terme de couleur a de dérivés, plus il est élémentaire.

Afin de comparer les noms de couleurs entre eux on a compté leurs dérivés ${ }^{5}$. Pour chaque dérivé le mot acquiert un point. Les dérivés ont été comptés selon le dictionnaire Le Petit Robert. En vue de standardisation, les formes non approuvées par ce dictionnaire n'ont pas été comptées, même si elles sont présentes dans d'autres sources (p.ex. orangeâtre).

Pour uniformiser les résultats obtenus de différents critères, on a recompté le nombre de dérivés en points finals selon l'algorithme suivant :

$0-4$ dérivés : 0 points

$5-8$ dérivés : 1 point

$9-12$ dérivés : 2 points

$13-15$ dérivés : 3 points

4. Il y en a 522 selon le site www.pourpre.com.

5. Les données pareilles ont déjà été rassemblées par Jean-Claude Lacroix en 1978. Lacroix l'a fait dans un autre but, mais, comme le remarque Kerttula, il se jette aux yeux que les noms de couleurs élémentaires ont plus de dérivés que les noms non élémentaires, cf. Kerttula (2002, p. 290). 
Tableau 1 La productivité dérivationnelle

\begin{tabular}{|c|c|c|c|c|c|c|}
\hline $\begin{array}{l}\text { nom de } \\
\text { couleur }\end{array}$ & verbes & adjectifs & noms & adverbes & $\begin{array}{c}\text { nombre de } \\
\text { dérivés }\end{array}$ & valeur \\
\hline noir & noircir & $\begin{array}{l}\text { noirâtre } \\
\text { noiraud }\end{array}$ & $\begin{array}{l}\text { noirceur } \\
\text { noircissement } \\
\text { noircisseur } \\
\text { noircissure } \\
\text { noire }\end{array}$ & - & 8 & 1 \\
\hline blanc & blanchir & $\begin{array}{l}\text { blanchâtre } \\
\text { blanchissant }\end{array}$ & $\begin{array}{l}\text { blancheur } \\
\text { blanchaille } \\
\text { blanchet } \\
\text { blanchiment } \\
\text { blanchissage } \\
\text { blanchissement } \\
\text { blanchisserie } \\
\text { blanchisseur } \\
\text { blanquette }\end{array}$ & - & 12 & 2 \\
\hline rouge & $\begin{array}{l}\text { rougir } \\
\text { rougeoyer }\end{array}$ & $\begin{array}{l}\text { rougeâtre } \\
\text { rougeaud } \\
\text { rougeoleux } \\
\text { rougeoyant } \\
\text { rougi } \\
\text { rougissant }\end{array}$ & $\begin{array}{l}\text { rougeur } \\
\text { rougissement } \\
\text { rougeoiement } \\
\text { rougeole } \\
\text { rouget }\end{array}$ & - & 13 & 3 \\
\hline jaune & jaunir & $\begin{array}{l}\text { jaunâtre } \\
\text { jaunasse } \\
\text { jaunissant } \\
\text { jaunet }\end{array}$ & $\begin{array}{l}\text { jaunet } \\
\text { jaunissage } \\
\text { jaunisse } \\
\text { jaunissement }\end{array}$ & - & 9 & 2 \\
\hline vert & $\begin{array}{l}\text { verdir } \\
\text { verdoyer }\end{array}$ & $\begin{array}{l}\text { verdâtre } \\
\text { verdissant } \\
\text { verdoyant } \\
\text { verdelet }\end{array}$ & $\begin{array}{l}\text { verdeur } \\
\text { verdissage } \\
\text { verdissement } \\
\text { verdage } \\
\text { verdet } \\
\text { verdier } \\
\text { verdoiement } \\
\text { verdure } \\
\end{array}$ & vertement & 15 & 3 \\
\hline bleu & bleuir & $\begin{array}{l}\text { bleuâtre } \\
\text { bleuissant } \\
\text { bleuté }\end{array}$ & $\begin{array}{l}\text { bleuet } \\
\text { bleuetière } \\
\text { bleueterie } \\
\text { bleuissement } \\
\text { bleusaille }\end{array}$ & - & 9 & 2 \\
\hline brun & brunir & $\begin{array}{l}\text { brunâtre } \\
\text { brunisseur }\end{array}$ & $\begin{array}{l}\text { brunissage } \\
\text { brunante }\end{array}$ & - & 11 & 2 \\
\hline
\end{tabular}

6. Les données ramassées par Lacroix diffèrent légèrement de ceux-ci. Pourtant il y a de grandes similarités dans l'ordre des mots entre ce tableau-ci, le tableau de Lacroix (d'après Kerttula, 2002, p. 290) et le tableau de Kerttula pour les termes anglais. Cela prouve l'universalité dans ce domaine, ainsi que la valeur méthodologique de ce critère. 


\begin{tabular}{|c|c|c|c|c|c|c|}
\hline & & & $\begin{array}{l}\text { brune } \\
\text { brunet } \\
\text { bruni } \\
\text { brunisseur } \\
\text { brunissoir } \\
\text { brunissure }\end{array}$ & - & & \\
\hline gris & $\begin{array}{l}\text { griser } \\
\text { grisailler } \\
\text { grisonner }\end{array}$ & $\begin{array}{l}\text { grisâtre } \\
\text { grisant } \\
\text { grisonnant }\end{array}$ & $\begin{array}{l}\text { grisaille } \\
\text { grisard } \\
\text { grisbi } \\
\text { grisé } \\
\text { griserie } \\
\text { griset } \\
\text { grisette } \\
\text { grisonnement } \\
\text { grison }\end{array}$ & - & 15 & 3 \\
\hline orange & orangé & - & - & - & 1 & 0 \\
\hline violet & - & - & - & - & 0 & 0 \\
\hline rose & $\begin{array}{l}\text { rosir } \\
\text { roser }\end{array}$ & rosé & $\begin{array}{l}\text { roseur } \\
\text { roselet } \\
\text { roséole } \\
\text { rosissement } \\
\text { rosage }\end{array}$ & & 8 & 1 \\
\hline pourpre & - & $\begin{array}{l}\text { pourpré } \\
\text { purpurin } \\
\text { purpurique }\end{array}$ & $\begin{array}{l}\text { purpura } \\
\text { purpurine }\end{array}$ & - & 5 & 1 \\
\hline $\begin{array}{l}\text { queue-de- } \\
\text { renard }\end{array}$ & - & - & - & - & 0 & 0 \\
\hline rubicond & - & - & - & - & 0 & 0 \\
\hline grenat & - & - & - & - & 0 & 0 \\
\hline
\end{tabular}

\subsection{La fréquence de l'usage}

La fréquence de l'usage du nom est un des critères les plus importants décidant de son caractère élémentaire. Pour mesurer la fréquence on s'est servi du Corpus français de l'Université de Leipzig (http://wortschatz.uni-leipzig.de/ws_fra/). Ce corpus est une base de données composée de près de 37 millions de phrases, soit environ 700 millions de mots.

On a additionné le nombre d'apparitions de toutes les formes possibles de chaque mot.

Puis, on a recalculé le nombre d'apparitions en points finals selon la formule suivante :

$$
\begin{aligned}
& <100 \text { apparitions : } 0 \text { points } \\
& 101-1000 \text { apparitions : } 1 \text { point } \\
& 1001-10000 \text { apparitions : } 2 \text { points }
\end{aligned}
$$


$10001-50000$ apparitions : 3 points

$50001-90000$ apparitions : 4 points

$>90000$ apparitions : 5 points

Tableau 2 La fréquence de l'usage

\begin{tabular}{|c|c|c|c|c|c|c|}
\hline $\begin{array}{l}\text { nom de } \\
\text { couleur }\end{array}$ & $\begin{array}{l}\text { sing. } \\
\text { masc. }\end{array}$ & $\begin{array}{l}\text { sing. } \\
\text { fem. }\end{array}$ & $\begin{array}{l}\text { plur. } \\
\text { masc. }\end{array}$ & $\begin{array}{l}\text { plur. } \\
\text { fem. }\end{array}$ & total & valeur \\
\hline noir & 57241 & 27215 & 13988 & 9162 & 107606 & 5 \\
\hline blanc & 47605 & 24653 & 14470 & 8781 & 95509 & 5 \\
\hline rouge & 66011 & - & 16766 & - & 82777 & 4 \\
\hline jaune & 23589 & - & 23589 & - & 47178 & 3 \\
\hline vert & 41908 & 10217 & 18393 & 4330 & 74848 & 4 \\
\hline bleu & 24136 & 10861 & 20261 & 3104 & 58362 & 4 \\
\hline brun & 4314 & 2849 & 1380 & 1181 & 9724 & 2 \\
\hline gris & 10848 & 5335 & - & 5335 & 21518 & 3 \\
\hline orange & 10736 & - & - & - & 10736 & 3 \\
\hline violet & 1981 & 1000 & 415 & 443 & 3839 & 2 \\
\hline rose & 13686 & - & 5332 & - & 19018 & 3 \\
\hline pourpre & 1487 & - & 366 & - & 1853 & 2 \\
\hline $\begin{array}{l}\text { queue-de- } \\
\text { renard }\end{array}$ & 2 & - & - & 0 & 2 & 0 \\
\hline rubicond & 29 & 6 & 2 & 4 & 41 & 0 \\
\hline grenat & 532 & - & - & - & 532 & 1 \\
\hline
\end{tabular}

\subsection{L'indépendance sémantique}

La signification d'un nom élémentaire ne peut pas être contenue dans celle d'un autre terme de couleur; cela implique la constatation que le nom de couleur élémentaire ne peut pas être l'hyponyme d'un autre terme désignant la couleur. De plus, le nom de couleur élémentaire possède sa signification de couleur comme le sens primaire. Ici, pour uniformiser la source des hiérarchies des significations, de nouveau on a pris en considération seulement les notes du Petit Robert. Pour mesurer ce critère on a utilisé l'échelle suivante :

Le sens primaire du terme c'est la couleur, le terme n'est pas l'hyponyme d'un autre : 3 points

La couleur est un sens primaire du terme, mais le mot est l'hyponyme d'un autre terme de couleur : 2 points

La couleur est le sens secondaire du terme : 1 point 
Tableau 3 L'indépendance sémantique

\begin{tabular}{|l|r|r|r|}
\hline nom de couleur & valeur 1 & valeur 2 & valeur 3 \\
\hline noir & - & - & 3 \\
\hline blanc & - & - & 3 \\
\hline rouge & - & - & 3 \\
\hline jaune & - & - & 3 \\
\hline vert & - & - & 3 \\
\hline bleu & - & - & 3 \\
\hline brun & - & - & 3 \\
\hline gris & - & - & 3 \\
\hline orange & 1 & - & - \\
\hline violet & - & - & 3 \\
\hline rose & 1 & - & - \\
\hline pourpre & 1 & - & - \\
\hline queue-de-renard & - & 2 & - \\
\hline rubicond & - & 2 & - \\
\hline grenat & 1 & - & - \\
\hline
\end{tabular}

\subsection{La structure morphologique}

Les noms de couleurs élémentaires n'ont pas la structure complexe du point de vue morphologique. Si le nom de couleurs se compose de plus que d'un morphème, on lui a supprimé le seul point possible à obtenir pour ce critère.

Tableau 4 La structure morphologique

\begin{tabular}{|l|r|r|}
\hline nom de couleur & valeur 1 & valeur 2 \\
\hline noir & & 1 \\
\hline blanc & & 1 \\
\hline rouge & & 1 \\
\hline jaune & & 1 \\
\hline vert & & 1 \\
\hline bleu & & 1 \\
\hline brun & & 1 \\
\hline gris & & 1 \\
\hline orange & & 1 \\
\hline violet & & 1 \\
\hline rose & & 1 \\
\hline pourpre & & 1 \\
\hline
\end{tabular}




\begin{tabular}{|l|r|r|}
\hline queue-de-renard & 0 & \\
\hline rubicond & & 1 \\
\hline grenat & & 1 \\
\hline
\end{tabular}

\subsection{L'accord}

Les noms de couleurs élémentaires se comportent d'une façon typique à la langue donnée. Dans le cas du français ils devraient s'accorder avec les substantifs en genre et en nombre. On n'a pas attribué les points aux adjectifs de couleurs invariables. Si ce n'est pas étonnant dans les cas du grenat et queue-de-renard, il faut remarquer que l'orange, considéré comme un nom élémentaire ne remplit cette condition non plus.

Tableau 5 L'accord

\begin{tabular}{|l|r|r|}
\hline nom de couleur & valeur 1 & valeur 2 \\
\hline noir & & 1 \\
\hline blanc & & 1 \\
\hline rouge & & 1 \\
\hline jaune & & 1 \\
\hline vert & & 1 \\
\hline bleu & & 1 \\
\hline brun & & 1 \\
\hline gris & & 1 \\
\hline orange & 0 & 1 \\
\hline violet & & 1 \\
\hline rose & & 1 \\
\hline pourpre & & \\
\hline queue-de-renard & & 1 \\
\hline rubicond & & \\
\hline grenat & & \\
\hline
\end{tabular}

\subsection{L'étendue sémantique}

Le nom élémentaire ne peut pas avoir un usage restreint à une classe limitée d'objets. Un tel nom doit avoir la possibilité de se référer à tout objet. Comme on voit, les noms de couleur étudiés ici remplissent le critère de l'extension sémantique, sauf le terme rubicond qui ne sert qu'à décrire la couleur de la peau ou du visage. C'est pourquoi on n'a pas attribué de points à ce terme. 
Tableau 6 L'étendue sémantique

\begin{tabular}{|l|r|r|}
\hline nom de couleur & valeur 1 & valeur 2 \\
\hline noir & & 1 \\
\hline blanc & & 1 \\
\hline rouge & & 1 \\
\hline jaune & & 1 \\
\hline vert & & 1 \\
\hline bleu & & 1 \\
\hline brun & 1 \\
\hline gris & & 1 \\
\hline orange & 1 \\
\hline violet & & 1 \\
\hline rose & & 1 \\
\hline pourpre & 1 \\
\hline queue-de-renard & & 1 \\
\hline rubicond & & 1 \\
\hline grenat & & \\
\hline
\end{tabular}

\section{Les résultats}

Les points que les noms de couleurs ont acquis pour leur élémentarité se répartissent d'une façon continue. La frontière entre les noms classifiés par Berlin et Kay comme élémentaires et les autres noms est floue (cf. Tableau 7).

Nettement, on peut distinguer le groupe des noms de couleurs élémentaires par excellence. Ce seront le noms qui ont au moins 10 points : blanc, rouge, vert, noir, bleu, gris, jaune et brun.

Ce qui est évident, on peut également distinguer le groupe des mots de couleurs qui sans doute ne peuvent pas être nommés élémentaires. Il s'agit des noms qui ont gagné au-dessous de 5 points. Comme on a pu supposer, grenat, queue-de-renard et rubicond appartiennent à cette catégorie.

Pourtant, ce qui est important, il y a toute une classe des noms qui sont difficiles à qualifier comme élémentaires ou non. C'est une question problématique car il s'agit entre autres des noms traditionnellement qualifiés d'élémentaires : rose, violet et orange. Le dernier a obtenu moins de points que pourpre, traditionnellement qualifié de non élémentaire.

L'analyse a démontré que certains noms de couleurs possèdent les traits décidant de leur caractère élémentaire et certains termes de couleurs en sont privés. Mais, ce qui est le plus significatif, on a pu voir que la frontière entre ces deux groupes n'est pas si nette que c'était présumé traditionnellement.

Enfin, il faut remarquer que les résultats sont pareils à ceux obtenus par Kerttula pour les noms de couleurs anglais, ce qui peut suggérer une certaine universalité dans ce domaine, au moins dans le cercle des langues indoeuropéennes. 
Tableau 7 Tableau récapitulatif

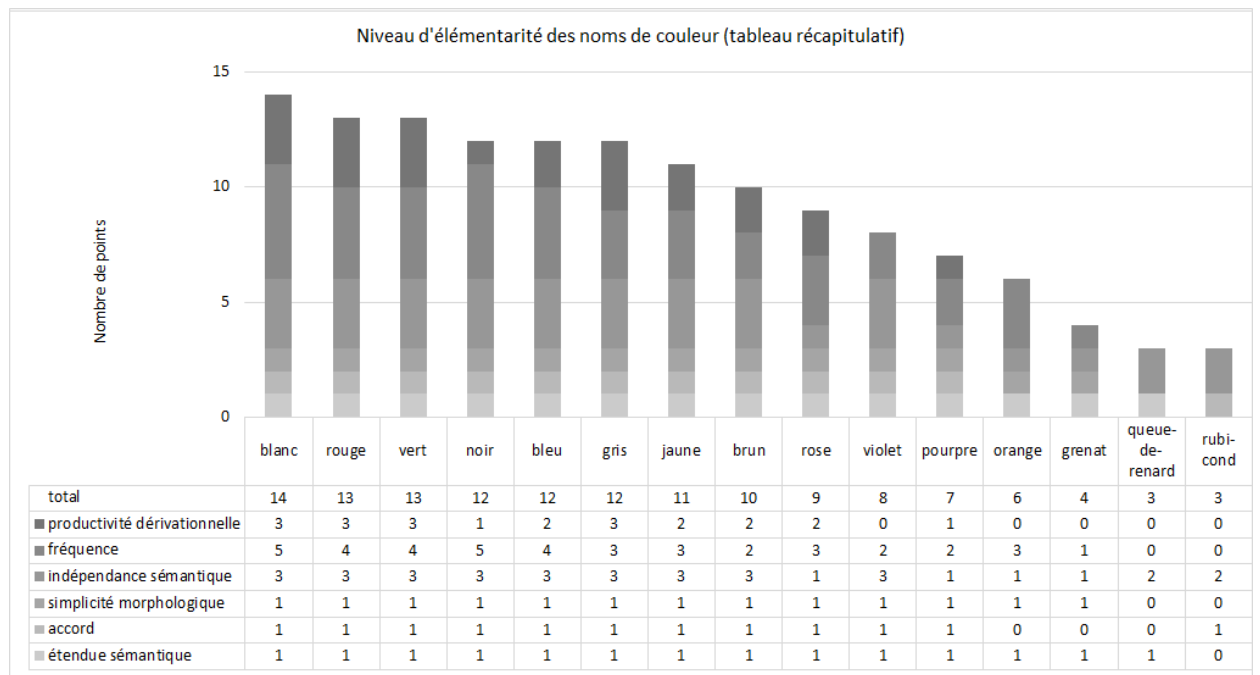

\section{Conclusion}

Le débat concernant la catégorisation linguistique des couleurs est plein de controverses. La question de relation entre la perception des couleurs et la langue a obtenu les réponses diverses. On peut trouver des solutions opposées : le déterminisme linguistique versus le déterminisme perceptuel. Apparemment, la réponse à cette question n'est pas si simple que ça. La recherche d'une simple corrélation entre le langage et la perception est condamnée à l'échec. Il semble que la langue et la réalité se forment mutuellement : elles s'influencent l'une l'autre.

La conclusion que l'on peut tirer de cette discussion durant deux siècles est la constatation que les termes de couleurs exigent la description à l'aide de la notion du prototype. Les deux conceptions de prototypes des couleurs, celle de Eleanor Rosch et celle de Anna Wierzbicka, bien que totalement différentes, ne se contredisent pas; elles se complètent même.

Pourtant, les régularités dans la dénomination des couleurs aperçues par Berlin et Kay sont toujours jugées vraies. Bien que cette conception de l'élémentarité exige l'interprétation traditionnelle de la catégorisation - en termes des conditions nécessaires et suffisantes, elle prédomine jusqu'à aujourd'hui.

Relativement peu d'auteurs rejettent la conception traditionnelle des noms élémentaires. Mais par exemple Seija Kerttula, avec son concept de l'élémentarité relative, constate que les noms de couleurs se trouvent à différentes étapes de leur développement. C'est pourquoi, ils peuvent être plus ou moins élémentaires mais il n'y a pas de frontière nette entre ces deux classes des noms de couleurs. Il semble qu'une telle conception de l'élémentarité soit plus proche de la réalité conceptuelle.

Premièrement, elle est plus conforme à la description à l'aide de la notion de prototype. Elle prouve qu'il y a quelques noms de couleurs prototypiques, ce sont les noms de couleurs par excellence, les exemplaires les plus parfaits des noms de couleurs; le reste de termes de couleurs étant plus ou moins éloigné du centre. 
Deuxièmement, la conception de l'élémentarité relative a résisté à des épreuves empiriques. Les critères qui décident du caractère élémentaire des termes de couleurs ont été utilisés pour vérifier l'hypothèse de l'élémentarité relative.

On a analysé une quinzaine de noms de couleurs français (entre autres tous les noms de couleurs élémentaires énumérés par Berlin et Kay) selon six critères préétablis : la productivité dérivationnelle, la fréquence de l'usage, l'indépendance sémantique, la simplicité morphologique, l'accord et l'étendue sémantique. Les résultats prouvent qu'il n'y a pas de frontière nette entre les noms de couleurs considérés comme élémentaires et ceux qui ne le sont pas.

La conception de l'élémentarité relative permet de faire une observation importante : les couleurs sont catégorisées comme concepts à structure prototypique. Il y a des noms de couleurs plus importants, plus proches du centre - les noms de couleurs élémentaires. Mais il y a également des noms plus et moins éloignés du centre. Les critères qui décident du caractère élémentaire des noms se répartissent d'une façon continue ce qui fait que certains noms se trouvent à la frontière entre les noms élémentaires et non élémentaires.

\section{Références}

Berlin, B., \& Kay, P. (1969). Basic color terms : Their universality and evolution. Berkeley : University of California Press.

Corpus français de l'Université de Leipzig. (n.d.). Retrieved from http://wortschatz. uni-leipzig.de/ws_fra/.

Czachorowska, M. (2009). Biały jak paulin, czarny jak miód prastary. O konkretyzacji prototypowej barwy białej i czarnej w historii języka polskiego. Studia Językoznawcze, $8,47-58$.

Delbecque, N. (2002). Linguistique cognitive. Comprendre comment fonctionne le langage. Bruxelles : De Boeck \& Larcier.

Heider, E. (1972). Universals in color naming and memory. Journal of Experimental Psychology, 93(1), 10-20. doi: 10.1037/h0032606.

Heider, E. (1973). On the internal structure of perceptual and semantic categories (pp. 111-144). dans : T. E Moore (Ed.), Cognitive development and the acquisition of language, pp. 111-144. New York, San Francisco, London : Academic Press.

Kerttula, S. (2002). English Colour Terms : Etymology, Chronology, and Relative Basicness. Helsinki : Société Néophilologique.

Kleiber, G. (2003). Semantyka prototypu. Kraków : Universitas.

Pietrzak-Porwisz, G. (2006). Semantyka bieli w języku polskim i szwedzkim. Studia Linguistica Universitatis Iagellonicae Cracoviensis, 123, 135-154.

Pinker, S. (2005). Tabula rasa. Spory o naturę ludzka. Gdańsk : Gdańskie Wydawnictwo Pedagogiczne.

Pourpre. (n.d). Retrieved from http://pourpre.com/.

Puppel, S. (2001). A Concise Guide to Psycholinguistics. Poznań : Wydawnictwo Poznańskie.

Taylor, J. R. (2001). Kategoryzacja w języku. Prototypy w teorii językoznawczej. Kraków: Universitas.

Tokarski, R. (2004). Semantyka barw we współczesnej polszczyźnie. Lublin: Wydawnictwo Uniwersytetu Marii Curie-Skłodowskiej. 
Waszakowa, K. (2009). Perspektywy badań porównawczych w zakresie semantyki leksykalnej w świetle językoznawczych teorii kognitywnych. LingVaria, 1(7), 49-64.

Wierzbicka, A. (1967). O języku dla wszystkich. Warszawa: Wiedza Powszechna.

Wierzbicka, A. (1999). Język - umyst - kultura. Warszawa: PWN.

Wierzbicka, A. (2006). Semantyka. Jednostki elementarne $i$ uniwersalne. Lublin: Wydawnictwo Uniwersytetu Marii Curie-Skłodowskiej.

Wittgenstein, L. (1998). Uwagi o kolorach. Warszawa: Spacja.

Zura, A. (2004). Prototypizacja i kategoryzacja w polu semantycznym barwy zielonej. Bohemistyka, 2, 105-120.

This is an Open Access article distributed under the terms of the Creative Commons Attribution 3.0 PL License (http://creativecommons.org/licenses/by/3.0/pl/), which permits redistribution, commercial and non-commercial, provided that the article is properly cited.

(C) The Author(s) 2014.

Publisher : Institute of Slavic Studies PAS \& University of Silesia in Katowice 